Infektiologie und Mikrobiologie

Redaktion: S. Schimanski

(Schwerpunkt Bakteriologie)/

Infectiology and Microbiology

(Focus Bacteriology)

\title{
Einsatz der Matrix-Assisted Laser Desorption/ionisation-Time of Flight Massenspektrometrie (MALDI-TOF MS) in der mikrobiologischen Routinediagnostik
}

\author{
Matrix-assisted laser desorption/ionisation time-of-flight mass spectrometry \\ (MALDI-TOF MS) in clinical microbiological routine diagnostics
}

\author{
Sören Schubert* und Andreas Wieser \\ Max von Pettenkofer-Institut für Hygiene und \\ Medizinische Mikrobiologie, München, Deutschland
}

\section{Zusammenfassung}

In der jüngsten Vergangenheit hat ein vollkommen neuartiger Ansatz zur Differenzierung von Bakterien und Pilzen die mikrobiologische Diagnostik erweitert: Die Identifikation von Keimen mittels MALDI-TOF Massenspektrometrie, die auf der Analyse ribosomaler Proteine beruht, ist bei vergleichbarer Validität der Untersuchungsergebnisse, wesentlich schneller als konventionelle, biochemische Differenzierungsverfahren. Neben der Identifizierung von Bakterienkolonien auf Festnährmedien können Bakterien und Sprosspilze aus positiven Blutkulturen oder direkt aus Urinproben analysiert werden. Derzeitige Weiterentwicklungen umfassen den Nachweis von $\beta$-Laktamase- und Carbapenemase-Aktivität der Bakterien sowie deren Genotypisierung unterhalb der Speziesebene.

Schlüsselwörter: Bakterienspezies; Blutkulturen; Differenzierung; MALDI-TOF; Sprosspilze.

\footnotetext{
Abstract

Very recently a novel method for differentiation of bacteria and fungi has been developed, that is, identification by means

*Korrespondenz: PD Dr. med. Sören Schubert, Max von Pettenkofer-Institut für Hygiene und Medizinische Mikrobiologie, Ludwig-Maximilians-Universität München, Marchioninistraße 17, 81377 München, Deutschland

Tel.: +49892180 78202

Fax: +4989218078294

E-Mail: schubert@med.uni-muenchen.de
}

of matrix-assisted laser desorption/ionisation time-of-flight mass spectrometry (MALDI-TOF MS). This differentiation relies on the exact measurement of species-specific protein spectra of ribosomal proteins. It is at least as accurate as conventional biochemical differentiation methods, but provides results within minutes. In addition to differentiation of bacteria and yeasts grown on agar plates, direct identification is feasible from positive blood cultures as well as from urine samples of patients suffering from urinary tract infections. Future developments of MALDI-TOF MS for clinical microbiological purposes include the detection of $\beta$-lactamase and carbapenemase activity as well as genotyping of bacteria below the species level.

Keywords: bacterial species; blood culture; differentiation; matrix-assisted laser desorption/ionisation time-of-flight (MALDI-TOF); yeast.

\section{Inhalte und Methodik der mikrobiologischen Diagnostik}

Das vornehmliche Ziel der medizinisch-mikrobiologischen Diagnostik ist es, in Patienten-Proben die für eine Erkrankung verantwortlichen Erreger (Bakterien, Pilze, Parasiten) zu identifizieren und - im Falle von Bakterien und Sprosspilzen - eine Resistenztestung für antimikrobielle Substanzen (Antibiogramm) zu bestimmen. Die eindeutige Speziesidentifikation von Krankheitserregern aus klinischen Materialien stellt dabei eine zentrale Aufgabe dar. Die klassische mikrobiologische Diagnostik beruht auf der Mikroskopie von Präparaten und der Kultivierung auf Nährmedien. Seit mehr als fünf Jahrzehnten werden zur Differenzierung von Bakterien und Pilzen vor allem biochemische Testverfahren eingesetzt, bei denen die Summe von Gattungs- und/oder Spezies-spezifischen Stoffwechseleigenschaften $\mathrm{zu}$ einem diagnostisch verwertbaren biochemischen Reaktionsprofil zusammengefügt wird. Wesentliche Nachteile dieses biochemischen Differenzierungsprinzips 
sind die langen Inkubationszeiten für die biochemischen Reaktionen (6 bis 12 Std.) und die Variabilität einzelner Stoffwechseleigenschaften sowie erhebliche laufende Kosten für Verbrauchsmaterialien. Ferner werden erhöhte Anforderungen an die Sachkenntnis und Erfahrung des durchführenden Laborpersonals gestellt, da ggf. eine Vordifferenzierung der Keime (z.B. durch Grampräparat, KliglerAgar und Katalase- oder Oxidase-Reaktion) notwendig ist. In den letzten Jahren ist ein vollkommen neuartiger Ansatz zur Differenzierung von Bakterien und Pilzen von der Entwicklung bis zur Routinetauglichkeit gekommen: Die Identifikation von Keimen mittels MALDI-TOF MS (MatrixAssisted-Laser-Desorption/Ionization-Time-of-FlightMass-Spectrometry - Matrix-unterstützte Laserdesorptions/ Ionisations-Flugzeit-Massenspektrometrie). Dieses massenspektrometrische Verfahren hat prinzipiell das Potenzial, die klassischen biochemischen Differenzierungsverfahren in der mikrobiologischen Diagnostik abzulösen, da es wesentliche Vorteile wie sehr kurze Analysenzeiten, einfache Bedienung und Automatisierbarkeit bei geringen Verbrauchskosten, sowie hohe Sensitivität und Spezifität in sich vereint [1].

\section{MALDI-TOF MS - methodische Grundlagen}

Die MALDI-TOF MS wird seit einigen Jahren mit Erfolg in der Proteom-Forschung verwendet, um die Masse von Proteinen und Peptiden zu bestimmen und in Kombination mit 1-D und 2-D Gelelektrophorese unbekannte Proteine zu identifizieren [2] (Abbildung 1). Die Technologie dient der Massenanalyse chemischer Verbindungen und stellt eine Kombination aus den Verfahren Matrix-unterstützte LaserDesorption/Ionisation (MALDI) und der Flugzeitanalyse (time of flight, TOF) dar.

Im Bereich der klinischen Chemie hat MALDI-TOF MS durch den Nachweis spezifischer Biomarker Einzug in die Diagnostik von Tumorerkrankungen, Morbus Alzheimer, rheumatoider Arthritis und Allergien gehalten [2]. Neben Proteinen und Peptiden können auch Nukleinsäuren mittels MALDI-TOF MS dargestellt werden.

Erste Versuche die massenspektrometrischen Verfahren zur Identifizierung von Mikroorganismen einzusetzen, wurden bereits 1975 durchgeführt [3]. Mit der Entwicklung der MALDI-TOF MS in den 1980er konnten erstmals relativ große Biomoleküle analysiert werden [4]. Daraufhin wurde begonnen, MALDI-TOF MS zur Identifizierung verschiedener Bakterienarten einzusetzen [1, 5, 6]. Diese ersten Untersuchungen waren durch schlechte Reproduzierbarkeit der Ergebnisse sowie durch die Abhängigkeit der Untersuchungsresultate von den Wachstumsbedingungen und den unterschiedlichen Nährmedien gekennzeichnet. Ein routinetauglicher Einsatz wurde erst möglich, als die Untersuchungen von niedermolekularen Massenbereichen $(<1000 \mathrm{Da})$ auf einen Massenbereich zwischen 2000 und $12,000 \mathrm{Da}$ verlagert wurden. Durch Verfeinerung der Methodik und der Software-gestützten Analytik kann die MALDI-TOF MS nun auch im Routinelaboratorium genutzt werden, um Mikroorganismen zu differenzieren.

Da die MALDI-TOF Massenspektrometrie ein sehr sensitives Verfahren darstellt, muss nur eine geringe Menge des Erregers (bei Bakterien reichen $10^{4}$ bis $10^{6} \mathrm{KBE}$ ) auf die MALDI-Probenplatte (Target) aufgebracht werden, um sie zu analysieren (Abbildung 2). Um diese Probe einzubetten, wird dazu etwa $1 \mu \mathrm{L}$ Matrix-Lösung aufgebracht und eingetrocknet. Dabei ko-kristallisiert die Matrix (Zimtsäure- oder Benzoesäurederivate) mit dem Erreger auf der Stahloberfläche der Targetplatte, die zwischen 12 und 160 Probenfelder trägt. Die beladenen Targetplatten werden in die Messkammer des Massenspektrometers eingeführt, in der kontinuierliches Hochvakuum herrscht. Um dieses aufrecht zu erhalten, sind Vakuumpumpen im Einsatz, die die beim Beladevorgang eingedrungene Luft wieder abpumpen. In der evakuierten Messkammer werden die einzelnen Probenfelder angesteuert und Teile der Probe durch mehrere kurze

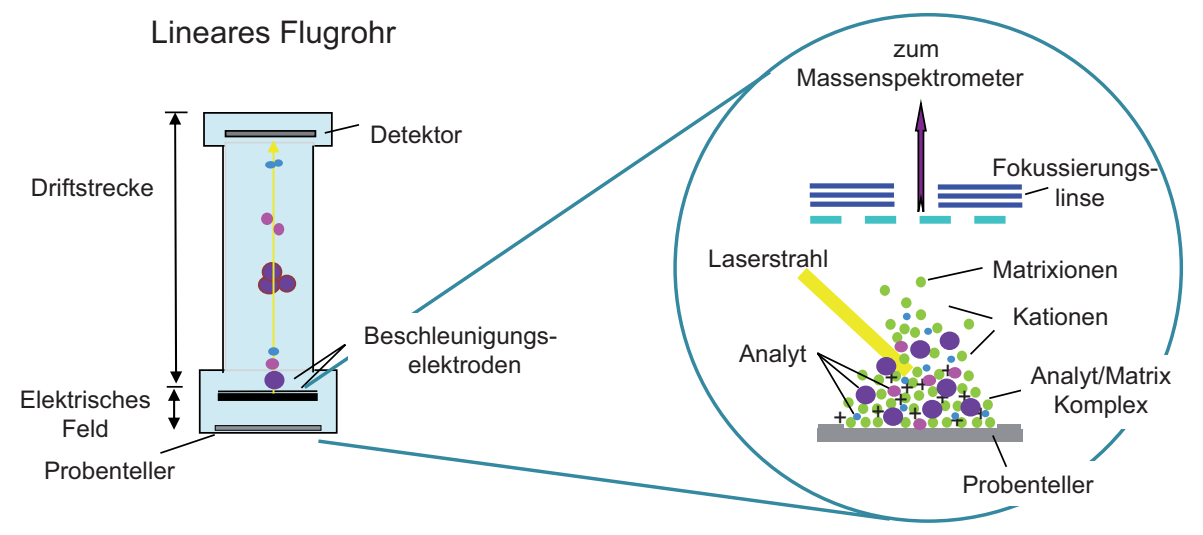

Abbildung 1 Prinzip der MALDI-TOF MS-basierten Identifikation von Proteinen und Peptiden.

Die Probe bzw. die Bakterien- oder Pilzkultur mit den darin befindlichen Proteinen (Analyte, dargestellt als blaue, rosa und lila Kugeln) wird zusammen mit der Matrix (grüne Kugeln) auf die Targetplatte gebracht. Nach Laserbeschuss werden die desorbierten Proteine im elektrischen Feld beschleunigt. Die Analyte trennen sich dann in der Driftstrecke gemäß ihrer Ladung und Masse auf. Durch die Bestimmung der Flugzeit der einzelnen Analyte entstehen charakteristische Massenspektren. 


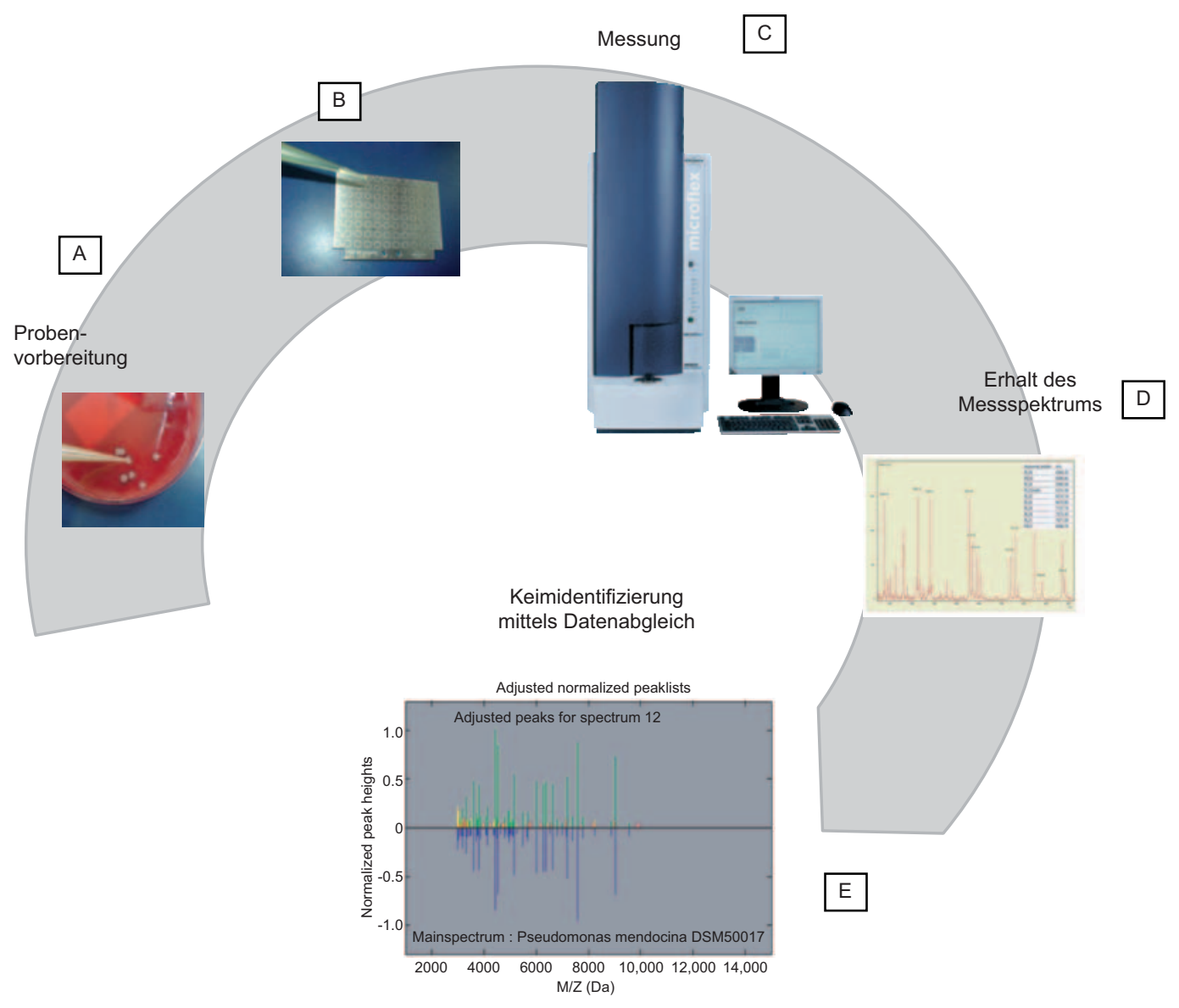

Abbildung 2 Arbeitsablauf.

(A) Abimpfen der Bakterienkolonien von der Agarplatte. (B) Aufbringen von einer Bakterienkolonie und Matrix auf das Target. (C) Messung im MALDI-TOF. (D) Erhalt des Messergebnisses als Spektrum. (E) Keimidentifizierung durch automatischen Abgleich des Spektrums mit der Datenbank.

Laserpulse mitsamt der Matrix explosionsartig verdampft. Dies bewirkt eine Ionisierung der Erregerproteine, die als Analyte im elektrischen Feld einer Hochspannungsquelle (10-30 KV) beschleunigt werden. Die Flugzeit bis zum Auftreffen der Analyten auf dem Detektor wird im Hochvakuum exakt gemessen. Sowohl der Ionisierungsgrad, als auch die Masse der Proteine bestimmen die Flugzeit der Proteine und ergeben bei jedem Erreger ein charakteristisches Spektrum, das eine Art massenspektrometrischen Fingerabdruck darstellt. Zur Identifizierung wird hauptsächlich das Massespektrum positiv geladener ribosomaler Proteine zwischen 2 und $12 \mathrm{KDa}$ benutzt, das sehr stabile und aussagekräftige Spektren liefert (Abbildung 3). Diese werden auch kaum von den Wachstumsbedingungen der Mikroorganismen beeinflusst.

Die vom Gerät gemessenen Spektren werden vollautomatisch mit Referenzdatenbanken abgeglichen, die eine große Zahl von Spektren medizinisch relevanter Isolate beinhalten. Der Datenbankabgleich kann durch neue Algorithmen automatisiert und nahezu in Echtzeit erfolgen [7, 8]. Dies ergibt eine sehr kurze Umsatzzeit bis zum positiven Ergebnis. Soll nur eine Probe gemessen werden, ist es möglich, ab dem Auftragen der Probe, binnen fünf bis sieben Minuten, eine
Speziesidentifizierung zu erhalten. Soll eine voll beladene Target-Platte mit 96 Isolaten mit Proben beschickt und gemessen werden, sind Zeiten von unter einer Stunde vom Auftrag der ersten Probe bis zur Messung des letzten Isolats möglich. Der Software-Vergleich des gemessenen Spektrums mit den hinterlegten Spektren in der Datenbank kann natürlich niemals genau identische Messwerte ergeben. Daher erzeugt die Software einen Zahlenwert (score-value), welcher über die Validität des Differenzierungsergebnisses Aufschluss gibt (Abbildung 4). Ferner gibt die Software das beste Ergebnis (best-Match) und auch weitere Ergebnisse aus, um die Plausibilitätskontrolle zu erleichtern (Abbildung 4). Die gemessenen Ergebnisse lassen sich über eine Computerverbindung direkt in die Labor-EDV einspeisen.

\section{Einsatz der MALDI-TOF Massenspektrometrie in der mikrobiologischen Diagnostik - Erregerdifferenzierung von Festnährmedien}

Vergleichsstudien zwischen MALDI-TOF MS-basierter und konventioneller, biochemischer Differenzierung von 


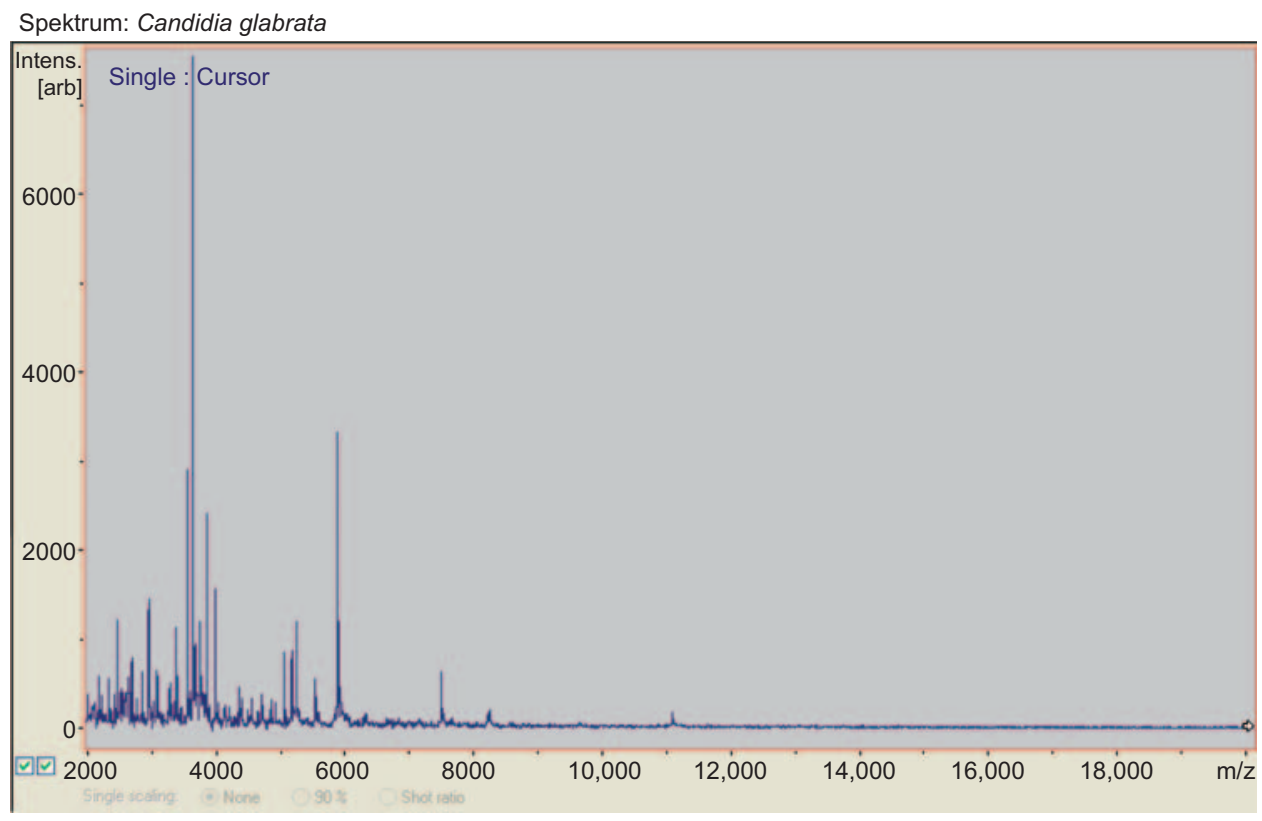

Abbildung 3 Massenspektrum einer Candida glabrata-Probe.

Im Messbereich von 4000 bis 8000 Da sind charakteristische Massenspitzen (,,peaks“) zu erkennen, die überwiegend ribosomale Proteine darstellen. Das Muster eines solchen Spektrums wird mit Hilfe der MALDI-Auswertungssoftware mit entsprechenden Datenbankeinträgen verglichen.

Bakterien und Sprosspilzen haben eine hohe Validität der MADLI-TOF Ergebnisse ergeben. Eine retrospektive Studie [9] an 1116 Isolaten vornehmlich im klinischmikrobiologischem Labor anzutreffenden Bakterien zeigte eine korrekte Identifikation durch MALDI-TOF MS in 95,2\% der Fälle. In einer weiteren kürzlich publizierten prospektiven Studie wurde gezeigt [10], dass von 1660 Bakterienisolaten aus 109 unterschiedlichen Spezies 84,1\% durch MALDI-TOF MS auf Speziesebene korrekt identifiziert wurden. Zu diesem Zeitpunkt noch fehlende Datenbank-Einträge wurden in verschiedenen Studien für nicht erfolgreiche Identifizierung verantwortlich gemacht.

Eigene Validierungsuntersuchungen des MALDI-TOF MS-Systems (Fa. Bruker Daltonik) unter Vergleich mit biochemischen Differenzierungssystemen (BD Phoenix ${ }^{\mathrm{TM}}$, API ${ }^{\circledR}$-bioMérieux) und ggf. anschließende Sequenzierungen der 16S-rDNA/28S-rDNA haben vergleichbare Ergebnisse erbracht. Bei 1200 prospektiv untersuchten Isolaten, darunter 370 Enterobacteriaceae, 300 Nonfermenter, 110 andere Gram-negative Bakterien, 320 Gram-positive Isolate und 100 Sprosspilze, wurde in 93,5\% der Fälle ein konkordantes Ergebnis zwischen MALDI-TOF MS und biochemischer Differenzierung erzielt. In 3\% der Fälle war die biochemische Differenzierung überlegen, bei $3,5 \%$ hatte die MALDITOF MS-Methode das exaktere Ergebnis geliefert, wenn die ebenfalls vorgenommene 16S-rDNA Sequenzierung als Goldstandard gewertet wurde. In vielen Fällen, in denen die MALDI-TOF MS-Analyse schlechtere Ergebnisse lieferte, waren lediglich die entsprechenden Referenzspektren der Isolate noch nicht in der Datenbank hinterlegt. Mittlerweile konnte durch das Erstellen von neuen Referenzspektren und entsprechende Erweiterungen der kommerziellen Datenbanken die diagnostische Lücke weitgehend geschlossen werden. Anders als bei den biochemischen Testsystemen handelt es sich beim MALDI-TOF MS-System um ein offenes, bei dem die Datenbank auch vom Anwender eigenständig erweitert werden kann. Durch entsprechende Ergänzungen der Referenz-Spektren der Datenbanken konnte eine nahezu 100\% Identifizierungsrate von Neisserien [11], Clostridien [12], Mycobakterien [13], Salmonellen [14], vergrünenden Streptokokken [15] und Helicobacter pylori [16] erreicht werden. Während das MALDI-TOF MS-System im Vergleich zu biochemischen Testsystemen im Bereich der Differenzierung von Enterobakterien, Staphylokokken und Streptokokken vergleichbar gute Ergebnisse liefert, zeigt es deutliche Vorteile bei der Differenzierung von Gram-positiven Stäbchen [17], Anaerobiern und einigen Nonfermentern [18, 19]. Insbesondere bei solchen Erregern, die sich durch lange Kultivierungszeiten auszeichnen, hat die MALDI-TOFbasierende Diagnostik eindeutige Zeitvorteile gebracht, die die Zeit bis zum Differenzierungsergebnis im Laboralltag drastisch reduziert haben.

MALDI-TOF MS wurde von verschiedenen Gruppen bereits zu Differenzierung von Spross- und Schimmelpilzen erfolgreich eingesetzt. Es konnte gezeigt werden, dass sich 96\% von 250 klinischen Candida-Isolate aus 15 Spezies mittels MALDI-TOF MS korrekt identifizieren ließen [20]. In zwei prospektiven Studien zur Identifikation von Sprosspilzen waren die Raten der korrekten Differenzierungsergebnisse vergleichbar hoch [21, 22]. Weit weniger Daten liegen für die Differenzierung von Schimmelpilzen, wie Aspergillus, Penicillium und Fusarium oder den Dermatophyten 
Ergebnis nach Datenbankvergleich

\begin{tabular}{|c|c|c|c|}
\hline $\begin{array}{c}\text { Rang } \\
\text { (Qualitat) }\end{array}$ & Ubereustiunmende Muster & $\begin{array}{l}\text { Bewertmings } \\
\text { zalli }\end{array}$ & $\begin{array}{l}\text { NCBI } \\
\text { Code }\end{array}$ \\
\hline $\begin{array}{c}1 \\
(+)\end{array}$ & Candida glabrata ATCC $90030 \mathrm{VML}$ & 2.187 & $\$ 478$ \\
\hline$\stackrel{2}{2}(++)$ & Candida glabrata DSM 70615 DSM & 2.132 & $\underline{5478}$ \\
\hline $\begin{array}{c}3 \\
(++)\end{array}$ & Candida glabrata ATCC 2001T THL & 2038 & 5478 \\
\hline $\begin{array}{c}4 \\
(++)\end{array}$ & Candida glabrata DSM 11950 DSM & 2.01 & $\$ 478$ \\
\hline $\begin{array}{c}5 \\
(+)\end{array}$ & Candida glabrata DSM 6425 DSM & 1.974 & $\$ 478$ \\
\hline $\begin{array}{c}6 \\
(-)\end{array}$ & Arthrobacter citreus IMET 10680T HKJ & 1.257 & $\underline{1670}$ \\
\hline $\begin{array}{c}7 \\
(-)\end{array}$ & Lactobacillus aglis DSM 20508 DSM & 1227 & $\underline{1601}$ \\
\hline $\begin{array}{l}8 \\
(-)\end{array}$ & Clostridum novyi A $1025 \_$NCTC 538 BOG & 1.225 & $\underline{1542}$ \\
\hline$\stackrel{9}{(-)}$ & Pseudomonas rhizosphaerae LMG 21640T HAM & 1.2 & $\underline{216142}$ \\
\hline $\begin{array}{l}10 \\
(-)\end{array}$ & Agromyces fucosus HKI 11529_DSMM 8597T HKJ & 1.195 & 41985 \\
\hline
\end{tabular}

Abbildung 4 Die Ergebnisse des Datenbank-Vergleichs können in tabellarischer Form dargestellt und automatisiert der Labor-EDV übermittelt werden.

Dabei werden die Ergebnisse mit einem Zahlenwert für die Validität der Differenzierung (,score“) versehen, um die Validität des Identifikationsergebnisses zu dokumentieren.

vor [23-27]. Die unterschiedlichen Zustandsformen der Schimmelpilze mit Myzel- und Konidien-Bildung und entsprechend unterschiedlichen Proteinmustern innerhalb eines Isolates erschweren die MALDI-TOF MS Identifikation [28]. Daher sind noch Anpassungen der Messung und deren Auswertung vorzunehmen, bevor die MALDI-TOFMessung von Schimmelpilzen vollständig für den Routinebetrieb geeignet ist. Da die MALDI-TOF-Differenzierung von angezüchteten Pilzen ausgeht und die Kultivierung von Dermatophyten sehr zeitaufwendig ist, bleibt abzuwarten, ob die MALDI-TOF Diagnostik in diesem Bereich großen Einzug hält. Viele Routinelaboratorien bieten eine Dermatophyten-Diagnostik nicht an, zumal die klinische Relevanz einer genauen Identifizierung in vielen Fällen gering ist (z.B. Fußpilz).

Anders als in den Anfängen der massenspektrometrischen Erregerdifferenzierung ist die heute eingesetzte, MALDI-TOF MS-basierte Untersuchung von der Art des Kulturmediums weitestgehend unabhängig. Auf Grund dessen können neben Kulturen aus Standardmedien wie Blutund Kochblutagar auch solche von Selektivnährböden wie McConkey-Agar oder XLD-Agar zur Differenzierung eingesetzt werden [6]. Da zur Identifikation der Isolate sehr wenig Kulturmaterial benötigt wird, reichen mitunter Kolonien von (Sub-)Kulturen aus, die bereits nach einigen Stunden der Inkubation entstehen. Auf diese Weise können Mischkulturen in Reinkulturen überführt und ggf. noch am selben Tag identifiziert werden. Die untersuchten Kolonien sollten allerdings möglichst frisch sein (nicht älter als 48 Std.), da mit zunehmendem Alter der Kolonien die ribosomalen Proteine der Bakterien degradiert werden. Dadurch fallen die MALDITOF MS-Spektren schwächer und weniger differenziert aus, was zu Schwierigkeiten beim Datenbank-Vergleich und zu schlechteren Differenzierungsergebnissen führen kann.

In den allermeisten Fällen kann die MALDI-TOF MSDifferenzierung der Bakterien ohne Probenvorbereitung durch einfaches Aufstreichen der Bakterienkolonie auf die Targetplatte erfolgen. Bei einigen Erregern, die eine stabile Zellwand aufweisen (z.B. bei Sprosspilzen) kann ein kurzer Extraktions-Schritt notwendig werden, um die ribosomalen Proteine der Analyse zugänglich zu machen. Hierzu wird z.B. die zu untersuchende Kolonie in $80 \%$-igem Ethanol aufgenommen, abzentrifugiert und danach in Ameisensäure und Acetonitril resuspendiert. Der resultierende Überstand wird nach Zentrifugation auf die Targetplatten aufgetragen. Durch diesen Extraktionsschritt lassen sich meist um ein Vielfaches klarere und vielschichtigere Spektren erzeugen, die dann eine erfolgreiche Datenbank-Suche ermöglichen. Nach einer Säuberung und Desinfektion mit $70 \%$ Ethanol kann die Targetplatte umgehend für weitere Messungen wieder eingesetzt werden.

Da die MALDI-TOF MS-Analyse bislang keine Möglichkeit für eine routinemäßige Antibiotika- oder AntimykotikaResistenzbestimmung der Isolate bietet, werden hierzu mittelfristig weiterhin herkömmliche manuelle, halbautomatische oder automatische Verfahren notwendig sein. Das MALDI-TOF MS-System zur Differenzierung lässt sich aber im Routinebetrieb problemlos mit den manuellen und automatischen Resistenzbestimmungsmethoden kombinieren. Eine Einbindung der MALDI-TOF MS-Analytik an das Labor-EDV System mittels uni- aber auch bidirektionaler Kommunikation ist bereits umsetzbar und in einigen größeren diagnostischen Zentren auch im Routinebetrieb verwirklicht. So können die Differenzierungsergebnisse online dem 
Automaten zur Resistenztestung übermittelt, und von diesem zur Interpretation der Resistenzen, bzw. der Resistenzmechanismen, genutzt werden.

\section{Einsatz der MALDI-TOF Massenspektrometrie in der mikrobiologischen Diagnostik - Direktidentifizierung von Erregern aus positiven Blutkulturen}

Schon frühzeitig wurde versucht, die MALDI-TOF MS zur Differenzierung von Bakterien und Pilzen direkt aus positiven Blutkulturen zu nutzen [29]. Das Problem bei dieser Applikation ist, dass große Mengen von Patientenproteinen in den Blutzellen den Nachweis der spezifischen Bakterienund Pilzproteine verschleiern. Mehrere Arbeitsgruppen haben in der Folge unterschiedliche Probenvorbereitungsprotokolle entwickelt, die entweder durch differenzielle Zentrifugation oder durch Zelllyse-Verfahren die Bakterien von den Wirtsproteinen trennen [30-32]. Die Studien zum Direktnachweis zeigen korrekte Identifikationsraten auf Spezies-Ebene von $75 \%-95 \%$ [33, 34]. Viele der Zentrifugations-Verfahren sind entweder zeitaufwendig und mit dem Routinearbeitsablauf im Diagnostik-Labor nur schwer vereinbar oder zu wenig effizient. Die Lysis-Verfahren werden derzeit favorisiert. Mit diesen schnell durchzuführenden Aufreinigung-Verfahren lassen sich innerhalb von 30 Minuten korrekte Identifikationen in $86 \%$ der Fälle erreichen. Bis auf die Schwierigkeiten der Abgrenzung von Streptococcus pneumoniae und Streptococcus mitis/oralis sind keine falschen Ergebnisse bei der direkten Blutkultur zu verzeichnen. Viele Studien fordern eine Adaptation der MALDI-TOF Score-Grenzwerte für die Validierung der Blutkultur-Differenzierungsergebnisse. Hintergrund ist, dass auch Messungen mit niedrigeren Score-Werten, die für die Messung von Bakterienkolonien als nicht ausreichend valide bewertet werden, bei der direkten Blutkultur-Identifikation korrekte Resultate liefern [32].

\section{Einsatz der MALDI-TOF Massenspektrometrie in der mikrobiologischen Diagnostik - Erregernachweis direkt aus Patientenproben (Urinproben)}

Die schnelle und einfache Identifizierung von Erregern mittels MALDI-TOF aus der Kultur kann nur eingeschränkt direkt auf Patientenproben übertragen werden. Dies liegt zuallererst an der Methode selbst, detektiert sie doch Erreger-Proteine und erzeugt daraus charakteristische Spektren. Proben, die direkt aus einem Patienten gewonnen werden, wie Sputum, Blut oder Liquor beinhalten jedoch sehr viel Protein des Patienten und nur vergleichsweise wenig vom Erreger. Somit ist es kaum möglich, die einzelnen Spektren zu trennen und diejenigen der Krankheitserreger herauszufiltern. Dazu kommt, dass in vielen Materialien eine mehr oder weniger komplexe Normalflora ebenfalls anzutreffen ist, deren Signale von den pathogenen Erregern getrennt werden müssten. In Materialien mit natürlicherweise geringem Proteingehalt, hoher Erregerlast und fehlender Normalflora wie etwa Urin, ist es jedoch möglich, Direktbestimmungen erfolgreich durchzuführen [35]. Dabei werden die Proben zuerst langsam zentrifugiert um Leukozyten zu pelletieren. Im zweiten Schritt erfolgt sodann eine schnelle Zentrifugation um die Erreger abzutrennen. Nach einem weiteren Waschschritt mit destilliertem Wasser sind die Erreger ausreichend aufgereinigt, um im MALDI-TOF MS erfolgreich analysiert werden zu können. Da der Harnwegsinfekt in den allermeisten Fällen nur durch einen prädominanten Erreger hervorgerufen wird, entstehen auch nur wenige Probleme mit gemischten Spektren. Mit solchen Protokollen konnten in größeren Studien aus Urin direkt korrekte Identifikationsraten von 91,8\% bis auf Speziesebene erreicht werden (Keimlast signifikant $>10^{5}$ $\mathrm{KBE} / \mathrm{mL}$ ) [35]. Damit stellt die Diagnostik mittels MALDITOF eine echte Alternative zu langwierigeren und teureren kulturbasierten Verfahren dar, speziell wenn im ambulanten Bereich gar keine Resistenztestung erfolgen soll.

\section{Einsatz der MALDI-TOF Massenspektrometrie in der mikrobiologischen Diagnostik - Vorteile, Möglichkeiten und Grenzen}

Die MALDI-TOF MS-basierte Erregeridentifizierung beruht wie oben dargelegt auf der Analyse von mehrheitlich ribosomalen Proteinen. Daher ähneln die Differenzierungsmuster denen der 16S-rDNA-Sequenzvergleiche. Auf Grund dessen ist die MALDI-TOF MS-Methode jedoch nicht in der Lage, genetisch sehr eng verwandte Mikroorganismen zu trennen. Klinisch bedeutsam ist dies etwa bei Shigella sp. und E. coli oder Streptococcus pneumoniae (Pneumokokken) und Vertretern der Streptococcus oralis/mitis-Gruppe. Hier ist auch weiterhin der Einsatz von biochemischen Ergänzungstests, Antigen-Nachweisen oder molekularen Methoden notwendig. Andererseits aber offenbart die MALDI-TOF MSAnalyse die Schwachpunkte der konventionellen, auf Stoffwechselreaktionen beruhenden Speziesdifferenzierung, deren Trennschärfe durch Verlust oder Zugewinn von Stoffwechseleigenschaften beschränkt sein kann. Dies ist der Grund, weshalb bei dem Vergleich von biochemischer mit molekularer und/oder MALDI-TOF MS-basierter Differenzierung in wenigen Fällen diskordante Ergebnisse resultieren, die auf den unterschiedlichen methodischen Ansätzen beruhen. Der enorme Zeitgewinn, die hohe Treffsicherheit und einfache Automatisierbarkeit der MALDI-TOF-MS-Analyse sind die wesentlichen Vorteile des Verfahrens im Laboralltag. Es kann ein einziges System zur Identifizierung der verschiedenen Bakteriengruppen und Sprosspilzen eingesetzt werden, eine Vorprüfung der Isolate muss nicht erfolgen. Da die Differenzierungsergebnisse bereits nach wenigen Minuten und am Tag der ersten Materialablesung, also 12 bis 24 Stunden nach Probeneingang, vorliegen, können den behandelnden Kollegen bereits viel früher wertvolle Zwischen- oder Vorbefunde übermittelt werden.

Ein weiterer Vorteil der MALDI-TOF MS-Methode liegt darin, dass Referenzspektren neuer Isolate sowohl als regelmäßig kommerziell erhältliche Datenbankupdates, als 
auch laborintern vom Nutzer selbst in das System integriert werden können. Denkbar wäre auch eine ,public domain“ in der MALDI-TOF-Benutzer Spektren hinterlegen können. So könnten bislang nicht ausreichend in der Datenbank repräsentierte Erregergruppen oder Spezies laufend ergänzt werden. Es muss nur sichergestellt werden, dass ausschließlich valide geprüfte Spektren tatsächlich Eingang in die Datenbanken finden, da sonst die Qualität der Identifikationen leiden kann.

Nachteilig bei der MALDI-TOF MS-Analyse ist bislang, dass der direkte Nachweis von Erregern aus Patientenproben sehr schwer möglich ist und daher fast immer eine Vorkultur erfolgen muss. Auch wenn es erste vielversprechende MALDI-TOF MS Untersuchungen zur Bestimmung bakterieller Resistenzen gegen $\beta$-Laktamantibiotika und Carbapeneme gibt, sind bislang im Routinebetrieb keine Aussagen zur Empfindlichkeit der Isolate gegenüber Antiinfektiva mittels MALDI-TOF MS-Untersuchung möglich. Es ist auch nicht zu erwarten, dass zukünftig mittels MALDI-TOF MS ein vollständiges Antibiotika-Resistenzprofil von Bakterienisolaten gegen unterschiedliche Substanzklassen erhalten werden kann. Zu unterschiedlich sind die Mechanismen und zu gering ist die Expression einzelner Resistenzfaktoren.

Die Anschaffungskosten für Routinediagnostik taugliche MALDI-TOF MS-Geräte inklusive Auswertungsrechner und Software mit integrierter Datenbank sind im Augenblick mit 180.000 bis 200.000 EUR noch relativ hoch. Im Gegenzug sind die laufenden Betriebskosten mit unter 50 Cent pro Analyse sehr gering. Zudem kann ein hoher Probendurchsatz mit nur einem Gerät erreicht werden. In naher Zukunft ist zu erwarten, dass durch verbesserte Halbleiter-Lasertechnik die Standzeiten steigen, sowie die möglichen Pulsfolgen schneller werden. Dies würde den Betrieb weiter beschleunigen und die laufenden Kosten senken.

Die Einführung von automatischen Reinigungssystemen der Ionen-Quelle (,Quellenheizung“) kann die Intervalle für routinemäßige Reinigungen verlängern und damit zu einer Reduktion von Wartungskosten sowie wartungsbedingten Ausfällen beitragen. In einer Studie wurden die Kosten der MALDI-TOF MS-Identifizierung auf ein Viertel der entsprechenden biochemischen Untersuchungen berechnet [10]. Hierbei haben die Autoren Verbrauchsmittel, Löhne der Beschäftigten und Geräteabschreibungen über eine 5-Jahresperiode einbezogen. Ähnliche Kostenreduktionen werden in einer weiteren Studie berichtet [36]. Mit Sicherheit muss die Kostenreduktion im jeweiligen Laborumfeld gesondert untersucht werden, Aussagen aus Studien sind nicht immer im vollen Umfang übertragbar.

\section{Zukünftige Applikationen der MALDI-TOF Massenspektrometrie in der mikrobiologischen Diagnostik}

Gegenwärtig werden weitere MALDI-TOFMS-Anwendungen für die klinisch-mikrobiologische Diagnostik entwickelt. Zum einen wird versucht, die Technologie zur Bestimmung ausgewählter Antibiotika-Resistenzen einzusetzen, wie etwa der Nachweis des Penicillin-bindenden Proteins PBP2a bei MRSA [37] oder von definierten $\beta$-Laktamase Typen bei Enterobacteriaceae. Zum anderen sind Entwicklungsarbeiten auf den Direktnachweis der Erreger aus Patientenproben ausgerichtet. Auch wird geprüft, ob das MALDI-TOF MS-Verfahren zur Genotyp-Analyse zukünftig zur Klärung epidemiologischer Fragestellungen eingesetzt werden kann. Dabei werden analog zu den bekannten RestriktionsFragment-Längen-Polymorphismus (RFLP)-Typisierungen Massenspektrumstypisierungen benutzt, die für Stämme ähnlich charakteristisch sind.

Zur Unterscheidung von Methicillin-resistenten Staphylococcus aureus-Isolaten (MRSA) von Methicillinsensiblen (MSSA) wurde die MALDI-TOF MS bereits eingesetzt $[38,39]$. Dabei wurde nicht der Nachweis des MRSA-typischen Penicillin-Bindungsprotein PBP2a geführt, sondern die Muster von Zellwandproteinen untersucht. So kann die Klonalität von S. aureus-Isolaten dargestellt werden, nicht alle Isolate eines ,typischen“ MRSA-Klons sind tatsächlich aber auch MRSA (z.B. Verlust des PBP2a). So überrascht nicht, dass mittlerweile auch widersprechende Daten veröffentlicht wurden [40, 41].

Ein vielversprechender Ansatz zur Identifikation von Resistenzen gegen $\beta$-Laktamantibiotikaist der MALDI-TOFMSbasierte Nachweis von Spaltprodukten der $\beta$-Laktamantibiotika und Carbapeneme durch den Einfluss entsprechender bakterieller Enzyme, der $\beta$-Laktamasen und Carbapenemasen. Durch den Einfluss dieser Enzyme wird der $\beta$-Laktam-, bzw. Penem-Ring im Molekül der jeweiligen Antibiotika hydrolytisch gespalten. Das entspricht der Addition eines $\mathrm{H}_{2} \mathrm{O}$-Moleküls und nachfolgender Decarboxylierung, bzw. Addition von Na. Nach 1 bis 3 Std. Inkubation des Antibiotikums mit einer Suspension des Teststammes lässt sich mittels MALDI-TOF MS die Veränderung der Masse des Antibiotika-Moleküls bestimmen und so die Wirkung der bakteriellen Enzyme am Testantibiotikum darstellen. Auf diesem Wege lassen sich bereits Resistenzen gegen verschiedene Penicilline, Cephalosporine und Carbapeneme, wie Meropenem und Imipenem, darstellen (ECCMID 2011, Poster, Sparbier et al.).

Weitere Entwicklungen gehen dahin mittels MALDITOF MS Bakterienisolate unterhalb der Speziesebene zu typisieren, um epidemiologische Aussagen zu einzelnen Patienten- oder Umweltisolaten treffen zu können. Dabei ist es Ziel, mittels diskreter Unterschiede der ermittelten Spektren eine individuelle Stammtypsierung durchzuführen, und damit zeit- und kostenaufwendige molekulare Untersuchungen (PCR, PFGE) überflüssig werden zu lassen.

Von großem Interesse vor allem für Laboratorien mit hohem Probendurchsatz ist die Entwicklung von Verarbeitungsstraßen. Neben dem Anlegen der Agarplatten und Flüssigmedien sind Bebrütungsvorgänge und DigitalPhotographie der bewachsenen Agarplatten zur Befundung Bestandteil dieser neuen Strategie. Mittlerweile haben mehrere Firmen die MALDI-TOF MS in dieses Konzept integriert, wobei durch Einsatz von Robotern die Kolonien von Agarplatten abgenommen und auf die MALDI-Targetplatten aufgebracht werden können. Hier ist die Entwicklung jedoch erst in ihren Anfängen. 


\section{Fazit}

Die derzeitig kommerziell angebotenen diagnostischen MALDI-TOF MS-Lösungen ermöglichen eine automatisierbare Keimdifferenzierung von kultivierten Bakterien- und Pilz-Spezies. Insgesamt sind von MALDT-TOF MS-basierten Verfahren nicht unerhebliche Veränderungen und Verbesserungen für die diagnostische Mikrobiologie zu erwarten Die klinisch-orientierte ärztliche Bewertung der erhobenen Daten durch einen erfahrenen Facharzt gewinnt in diesem Prozess einen sehr hohen Stellenwert.

\section{Danksagung}

Unsere Arbeiten zur Weiterentwicklung der diagnostischen MALDI-TOF MS-Technologie werden durch die Bayerische Forschungsstiftung unterstützt (Forschungsverbund FORPROTECT - Infektionsprotektion durch neue Diagnostikverfahren und Therapieansätze).

\section{Literatur}

1. Claydon MA, Davey SN, Edwards-Jones V, Gordon DB. The rapid identification of intact microorganisms using mass spectrometry. Nat Biotechnol 1996;14:1584-6.

2. Marvin LF, Roberts MA, Fay LB. Matrix-assisted laser desorption/ionization time-of-flight mass spectrometry in clinical chemistry. Clin Chim Acta 2003;337:11-21.

3. Anhalt JP, Fenselau C. Identification of bacteria using mass spectrometry. Anal Chem 1975;47:219-25.

4. Hillenkamp F, Karas M. Mass spectrometry of peptides and proteins by matrix assisted ultraviolet laser desorption/ionization. Methods Enzymol 1990;193:280-95.

5. Demirev PA, Ho YP, Ryzhov V, Fenselau C. Microorganism identification by mass spectrometry and protein database searches. Anal Chem 1999;71:2732-8.

6. Fenselau C, Demirev PA. Characterization of intact microorganisms by MALDI mass spectrometry. Mass Spectrom Rev 2001;20:157-71.

7. Jarman KH, Cebula ST, Saenz AJ, Petersen CE, Valentine NB, Kingsley MT, et al. An algorithm for automated bacterial identification using matrix-assisted laser desorption/ionization mass spectrometry. Anal Chem 2000;72:1217-23.

8. Sauer S, Freiwald A, Maier T, Kube M, Reinhardt R, Kostrzewa $\mathrm{M}$, et al. Classification and identification of bacteria by mass spectrometry and computational analysis. PLoS ONE 2008;3: e2843.

9. Eigner U, Holfelder M, Oberdorfer K, Betz-Wild U, Bertsch D, Fahr AM. Performance of a matrix-assisted laser desorption ionization-time-of-flight mass spectrometry system for the identification of bacterial isolates in the clinical routine laboratory. Clin Lab 2009;55:289-96.

10. Seng P, Drancourt M, Gouriet F, La SB, Fournier PE, Rolain JM, et al. Ongoing revolution in bacteriology: routine identification of bacteria by matrix-assisted laser desorption ionization time-of-flight mass spectrometry. Clin Infect Dis 2009;49: 543-51.

11. Ilina EN, Borovskaya AD, Malakhova MM, Vereshchagin VA, Kubanova AA, Kruglov AN, et al. Direct bacterial profiling by matrix-assisted laser desorption - ionization time-of-flight mass spectrometry for identification of pathogenic neisseria. J Mol Diagn 2009;11:75-86.

12. Grosse-Herrenthey A, Maier T, Gessler F, Schaumann R, Bohnel $\mathrm{H}$, Kostrzewa $\mathrm{M}$, et al. Challenging the problem of clostridial identification with matrix-assisted laser desorption and ionization-time-of-flight mass spectrometry (MALDI-TOF MS). Anaerobe 2008;14:242-9.

13. Pignone M, Greth KM, Cooper J, Emerson D, Tang J. Identification of mycobacteria by matrix-assisted laser desorption ionization-time-of-flight mass spectrometry. J Clin Microbiol 2006;44:1963-70.

14. Dieckmann R, Helmuth R, Erhard M, Malorny B. Rapid classification and identification of salmonellae at the species and subspecies levels by whole-cell matrix-assisted laser desorption ionization-time of flight mass spectrometry. Appl Environ Microbiol 2008;74:7767-78.

15. Friedrichs C, Rodloff AC, Chhatwal GS, Schellenberger W, Eschrich K. Rapid identification of viridans streptococci by mass spectrometric discrimination. J Clin Microbiol 2007;45:2392-7.

16. Ilina EN, Borovskaya AD, Serebryakova MV, Chelysheva VV, Momynaliev KT, Maier T, et al. Application of matrix-assisted laser desorption/ionization time-of-flight mass spectrometry for the study of Helicobacter pylori. Rapid Commun Mass Spectrom 2010;24:328-34.

17. Barbuddhe SB, Maier T, Schwarz G, Kostrzewa M, Hof H, Domann E, et al. Rapid identification and typing of listeria species by matrix-assisted laser desorption ionization-time of flight mass spectrometry. Appl Environ Microbiol 2008;74:5402-7.

18. Mellmann A, Cloud J, Maier T, Keckevoet U, Ramminger I, Iwen $\mathrm{P}$, et al. Evaluation of matrix-assisted laser desorption ionizationtime-of-flight mass spectrometry in comparison to $16 \mathrm{~S}$ rRNA gene sequencing for species identification of nonfermenting bacteria. J Clin Microbiol 2008;46:1946-54.

19. Vanlaere E, Sergeant K, Dawyndt P, Kallow W, Erhard M, Sutton H, et al. Matrix-assisted laser desorption ionisationtime-of of-flight mass spectrometry of intact cells allows rapid identification of Burkholderia cepacia complex. J Microbiol Methods 2008;75:279-86.

20. Marklein G, Josten M, Klanke U, Muller E, Horre R, Maier T, et al. Matrix-assisted laser desorption ionization-time of flight mass spectrometry for fast and reliable identification of clinical yeast isolates. J Clin Microbiol 2009;47:2912-7.

21. Bizzini A, Durussel C, Bille J, Greub G, Prod'hom G. Performance of matrix-assisted laser desorption ionization-time of flight mass spectrometry for identification of bacterial strains routinely isolated in a clinical microbiology laboratory. J Clin Microbiol 2010;48:1549-54.

22. van Veen SQ, Claas EC, Kuijper EJ. High-throughput identification of bacteria and yeast by matrix-assisted laser desorption ionization-time of flight mass spectrometry in conventional medical microbiology laboratories. J Clin Microbiol 2010;48:900-7.

23. Amiri-Eliasi B, Fenselau C. Characterization of protein biomarkers desorbed by MALDI from whole fungal cells. Anal Chem 2001;73:5228-31.

24. Erhard M, Hipler UC, Burmester A, Brakhage AA, Wostemeyer J. Identification of dermatophyte species causing onychomycosis and tinea pedis by MALDI-TOF mass spectrometry. Exp Dermatol 2008;17:356-61.

25. Hettick JM, Green BJ, Buskirk AD, Kashon ML, Slaven JE, Janotka E, et al. Discrimination of Aspergillus isolates at the species and strain level by matrix-assisted laser desorption/ 
ionization time-of-flight mass spectrometry fingerprinting. Anal Biochem 2008;380:276-81.

26. Hettick JM, Green BJ, Buskirk AD, Kashon ML, Slaven JE, Janotka E, et al. Discrimination of Penicillium isolates by matrix-assisted laser desorption/ionization time-of-flight mass spectrometry fingerprinting. Rapid Commun Mass Spectrom 2008;22:2555-60.

27. Marinach-Patrice C, Lethuillier A, Marly A, Brossas JY, Gene J, Symoens F, et al. Use of mass spectrometry to identify clinical Fusarium isolates. Clin Microbiol Infect 2009;15:634-42.

28. Santos C, Paterson RR, Venancio A, Lima N. Filamentous fungal characterizations by matrix-assisted laser desorption/ ionization time-of-flight mass spectrometry. J Appl Microbiol 2010;108:375-85.

29. La SB, Raoult D. Direct identification of bacteria in positive blood culture bottles by matrix-assisted laser desorption ionisation time-of-flight mass spectrometry. PLoS One 2009;4:e8041.

30. Christner M, Rohde H, Wolters M, Sobottka I, Wegscheider K, Aepfelbacher M. Rapid identification of bacteria from positive blood culture bottles by use of matrix-assisted laser desorptionionization time of flight mass spectrometry fingerprinting. J Clin Microbiol 2010;48:1584-91.

31. Ferroni A, Suarez S, Beretti JL, Dauphin B, Bille E, Meyer J, et al. Real-time identification of bacteria and Candida species in positive blood culture broths by matrix-assisted laser desorption ionization-time of flight mass spectrometry. J Clin Microbiol 2010;48:1542-8.

32. Stevenson LG, Drake SK, Murray PR. Rapid identification of bacteria in positive blood culture broths by matrix-assisted laser desorption ionization-time of flight mass spectrometry. J Clin Microbiol 2010;48:444-7.

33. Ferreira L, Sanchez-Juanes I, Porras-Guerra I, Garcia-Garcia MI, Garcia-Sanchez JE, Gonzalez-Buitrago JM, et al. Microorganisms direct identification from blood culture by MALDI-TOF mass spectrometry. Clin Microbiol Infect 2011;17:546-51.
34. Prod'hom G, Bizzini A, Durussel C, Bille J, Greub G. Matrixassisted laser desorption ionization-time of flight mass spectrometry for direct bacterial identification from positive blood culture pellets. J Clin Microbiol 2010;48:1481-3.

35. Ferreira L, Sanchez-Juanes F, Gonzalez-Avila M, CembreroFucinos D, Herrero-Hernandez A, Gonzalez-Buitrago JM, et al. Direct identification of urinary tract pathogens from urine samples by matrix-assisted laser desorption ionization-time of flight mass spectrometry. J Clin Microbiol 2010;48:2110-5.

36. Cherkaoui A, Hibbs J, Emonet S, Tangomo M, Girard M, Francois $\mathrm{P}$, et al. Comparison of two matrix-assisted laser desorption ionization-time of flight mass spectrometry methods with conventional phenotypic identification for routine identification of bacteria to the species level. J Clin Microbiol 2010;48:1169-75.

37. Jackson KA, Edwards-Jones V, Sutton CW, Fox AJ. Optimisation of intact cell MALDI method for fingerprinting of methicillinresistant Staphylococcus aureus. J Microbiol Methods 2005;62: 273-84.

38. Edwards-Jones V, Claydon MA, Evason DJ, Walker J, Fox AJ, Gordon DB. Rapid discrimination between methicillin-sensitive and methicillin-resistant Staphylococcus aureus by intact cell mass spectrometry. J Med Microbiol 2000;49:295-300.

39. Majcherczyk PA, McKenna T, Moreillon P, Vaudaux P. The discriminatory power of MALDI-TOF mass spectrometry to differentiate between isogenic teicoplanin-susceptible and teicoplanin-resistant strains of methicillin-resistant Staphylococcus aureus. FEMS Microbiol Lett 2006;255:233-9.

40. Bernardo K, Pakulat N, Macht M, Krut O, Seifert H, Fleer S, et al. Identification and discrimination of Staphylococcus aureus strains using matrix-assisted laser desorption/ionization-time of flight mass spectrometry. Proteomics 2002;2:747-53.

41. Du Z, Yang R, Guo Z, Song Y, Wang J. Identification of Staphylococcus aureus and determination of its methicillin resistance by matrix-assisted laser desorption/ionization time-offlight mass spectrometry. Anal Chem 2002;74:5487-91. 